\title{
MXD1 localizes in the nucleolus, binds UBF and impairs rRNA synthesis
}

\author{
Maria del Carmen Lafita-Navarro ${ }^{1,3}$, Rosa Blanco ${ }^{1}$, Jorge Mata-Garrido², Judit \\ Liaño-Pons ${ }^{1}$, Olga Tapia ${ }^{2}$, Lucía García-Gutiérrez ${ }^{1}$, Eva García-Alegría ${ }^{1,4}$, María T. \\ Berciano², Miguel Lafarga², Javier León ${ }^{1}$ \\ ${ }^{1}$ Instituto de Biomedicina y Biotecnología de Cantabria (IBBTEC), CSIC-Universidad de Cantabria, and Department of \\ Molecular Biology, University of Cantabria, Santander, Spain \\ ${ }^{2}$ Department of Anatomy and Cell Biology and Centro de Investigación en Red sobre Enfermedades Neurodegenerativas \\ (CIBERNED), University of Cantabria-IDIVAL, Santander, Spain \\ ${ }^{3}$ Present address: Department of Cell Biology, UT Southwestern Medical Center, Dallas, Texas, USA \\ ${ }^{4}$ Present address: Stem Cell Hematopoiesis Group, Cancer Research UK Manchester Institute, University of Manchester, \\ Manchester, United Kingdom
}

Correspondence to: Javier León, email: leonj@unican.es

Keywords: $M X D 1, U B F$, nucleolus, pre-rRNA, transcription regulation

Received: June 20, $2016 \quad$ Accepted: August 26, $2016 \quad$ Published: August 31, 2016

\section{ABSTRACT}

MXD1 is a protein that interacts with MAX, to form a repressive transcription factor. MXD1-MAX binds E-boxes. MXD1-MAX antagonizes the transcriptional activity of the MYC oncoprotein in most models. It has been reported that MYC overexpression leads to augmented RNA synthesis and ribosome biogenesis, which is a relevant activity in MYC-mediated tumorigenesis. Here we describe that MXD1, but not MYC or MNT, localizes to the nucleolus in a wide array of cell lines derived from different tissues (carcinoma, leukemia) as well as in embryonic stem cells. MXD1 also localizes in the nucleolus of primary tissue cells as neurons and Sertoli cells. The nucleolar localization of MXD1 was confirmed by co-localization with UBF. Co-immunoprecipitation experiments showed that MXD1 interacted with UBF and proximity ligase assays revealed that this interaction takes place in the nucleolus. Furthermore, chromatin immunoprecipitation assays showed that MXD1 was bound in the transcribed rDNA chromatin, where it co-localizes with UBF, but also in the ribosomal intergenic regions. The MXD1 involvement in rRNA synthesis was also suggested by the nucleolar segregation upon rRNA synthesis inhibition by actinomycin $D$. Silencing of MXD1 with siRNAs resulted in increased synthesis of pre-rRNA while enforced MXD1 expression reduces it. The results suggest a new role for MXD1, which is the control of ribosome biogenesis. This new MXD1 function would be important to curb MYC activity in tumor cells.

\section{INTRODUCTION}

MXD1 (also known as MAD1) is a transcription factor belonging to the MXD family of proteins [1]. The MXD family (composed of MXD1, MXI1/MXD2, MXD3, MXD4 and MNT) is part of the MYC-MAXMXD network of transcription factors. These factors contain the b-HLH-LZ domain by which they bind to E-boxes in the promoter of their target genes to regulate their transcription [2-4].Whereas MYC-MAX activates transcription, MXD1-MAX represses transcription forming complexes with histone deacetylases. Therefore,
MXD1 acts as antagonist of MYC transcriptional activity [5-7]. Conversely to MYC, MXD1 is readily expressed in resting and differentiated cells $[6,8,9]$. Moreover, MXD1 inhibits cell proliferation and antagonize MYC transforming activity [10-12].

The nucleolus is the nuclear factory for rRNA synthesis, posttranscriptional processing of pre-rRNA and assembly of preribosomal particles. It is organized around clusters of tandem repeats of RNA genes (rDNA) that encode the rRNAs 28S, $18 \mathrm{~S}$ and 5.8S. The nucleolar structure, composed of fibrillar centers (FCs), dense fibrillar component (DFC) and granular component 
(GC), reflects the sequential steps of ribosome biogenesis [13-15]. The FC concentrates components of the transcription machinery, such as RNA polymerase I and UBF (Upstream Binding Factor), an essential transcription factor of ribosomal genes [16]. The DFC partially surrounds FCs and contains transcriptionally active ribosomal genes and numerous RNA-binding proteins. RNA pol I transcription takes place in the FCs and in the DFC, with further posttranscriptional processing of pre-rRNA occurring within the DFC to give rise to the mature rRNAs $[17,18]$. The GC is where the $40 \mathrm{~S}$ and $60 \mathrm{~S}$ ribosome subunits are assembled [19, 20].

MXD1 was described as nuclear in fibroblasts [1] and quiescent keratinocytes [21], but the subcellular localization of endogenous MXD1 is unknown for most cell types. Here we report that MXD1 localizes in the cell nucleolus and describe an interaction of MXD1 with UBF. In addition, we show that MXD1 binds to rDNA and regulates rDNA transcription.

\section{RESULTS}

\section{MXD1 localization in the nucleolus}

We performed immunofluorescence studies in proliferating embryonic stem cells HS181, mesenchymal stem cells MSC-3H, myeloid leukemia K562 cells, embryonic kidney HEK293T cells, and cervical cancer HeLa cells. Although it is reported that MXD1 is mostly expressed in quiescent cells or differentiated cells, we found low but detectable MXD1 protein levels in proliferating cells of the above cell lines (Figure 1A), although serum deprivation resulted in increased MXD1 expression, as described [22] (Figure 1A). We chose some of these cell lines with higher MXD1 expression to study its subcellular localization. As shown in Figure 1B, MXD1 was localized in the nucleus in all cell lines but, surprisingly, it was also concentrated in the nucleolus in a relevant proportion of cells $(70 \%$ to $100 \%$ depending on the cell line). The nucleolar distribution of MXD1 was confirmed by its co-localization with UBF (Figure 1B), a nucleolar marker of FCs $[13,23]$. The nucleolar localization for MXD1 is so far unreported and we set out to confirm this. Analysis of the fluorescence intensity profiles of UBF and MXD1 in cell lines and neurons demonstrated this co-localization (Supplementary Figure S1). We also asked if the subnuclear localization of MXD1 observed in cells in culture also occurred in primary tissue cells. We analysed by immunofluorescence Sertoli and germinal cells from rat seminiferous tube and rat sensory ganglion neurons. MXD1 was concentrated in the nucleolus, with a weaker nucleoplasmic signal, in post-mitotic Sertoli cells and neurons (Figure 2A). The nucleolar localization of MXD1 was confirmed in preparations co-stained with propidium iodide (Figure 2A), and its preferential concentration in FCs of neurons was demonstrated by co-localization with UBF (Figure 1A, lower panel). Moreover, immunogold electron microscopy confirmed the localization of MXD1 in FCs and associated DFC of the neuronal nucleolus (Figure 2B). To further confirm this subnuclear distribution, we transfected HeLa cells with a GFP-MXD1 construct. As controls, we also transfected a GFP-MNT and GFP constructs. Following $24 \mathrm{~h}$ of transfection, the transfected GFP-MXD1 protein was diffusely distributed in the nucleoplasm, but concentrated in the nucleolus (Figure 3A). The nucleolar localization of exogenous GFP-MXD1 was confirmed by immunofluorescence with the anti-UBF antibody, showing the co-localization of GFP and UBF signals (Figure 3B).

We next studied whether the nucleolar distribution of MXD1 was extensible to MYC and another member of the MXD family as MNT. In sharp contrast to MXD1, both MYC and MNT were present in the nucleoplasm but excluded from the UBF-immunolabeled nucleoli in most cells (Figure 4). Fluorescence intensity line profiles confirmed the absence of MYC and MNT in the nucleolus (Supplementary Figure S2). Moreover, overexpressed MNT did not accumulate in the nucleolus, as shown in HeLa cells transfected with a GFP-MNT construct (Figure 3A).

The co-localization of MXD1 and UBF in the FCs opened the possibility that MXD1 might be regulating rDNA transcription. To test this, we treated HS181 and MSC-3H cells with actinomycin D at low concentration (50 ng/ml for $1 \mathrm{~h}$ ), and performed co-immunostaining for MXD1 and UBF. Under these conditions, actinomycin D inhibits RNA pol I but not RNA pol II and RNA pol III [24, 25]. We also treated rats systemically with actinomycin (300 $\mu \mathrm{g} / \mathrm{kg}$ for $3 \mathrm{~h})$ and analysed the nucleolar reorganization in sensory neurons from trigeminal ganglia. Interestingly, we found that RNA pol I inhibition interfered with the nucleolar targeting of MXD1 in all cases, as a MXD1-free nucleolar area appeared after treatment with actinomycin D, meanwhile the nucleoplasmic signal of MXD1 remained unchanged (Figure 5). In MSC-3H cells and sensory neurons, MXD1 was segregated at the nucleolar periphery, forming typical perinucleolar caps or rings in confocal images (Figure 5) [26]. This effect was associated with disruption of FCs and peripheral segregation of the UBF, suggesting that MXD1 localization in FCs correlates with ongoing rRNA synthesis and raising a possible role of this transcription factor in the regulation of rDNA transcription.

\section{Interaction between MXD1 and UBF}

As UBF and MXD1 co-localized in the FCs of nucleolus, we asked whether MXD1 and UBF interacted in the nucleolus. We first performed immunoprecipitation assays in HeLa cells. The cells were deprived from serum for $48 \mathrm{~h}$ to augment MXD1 expression and the lysates were immunoprecipitated with anti-UBF antibodies. 
A
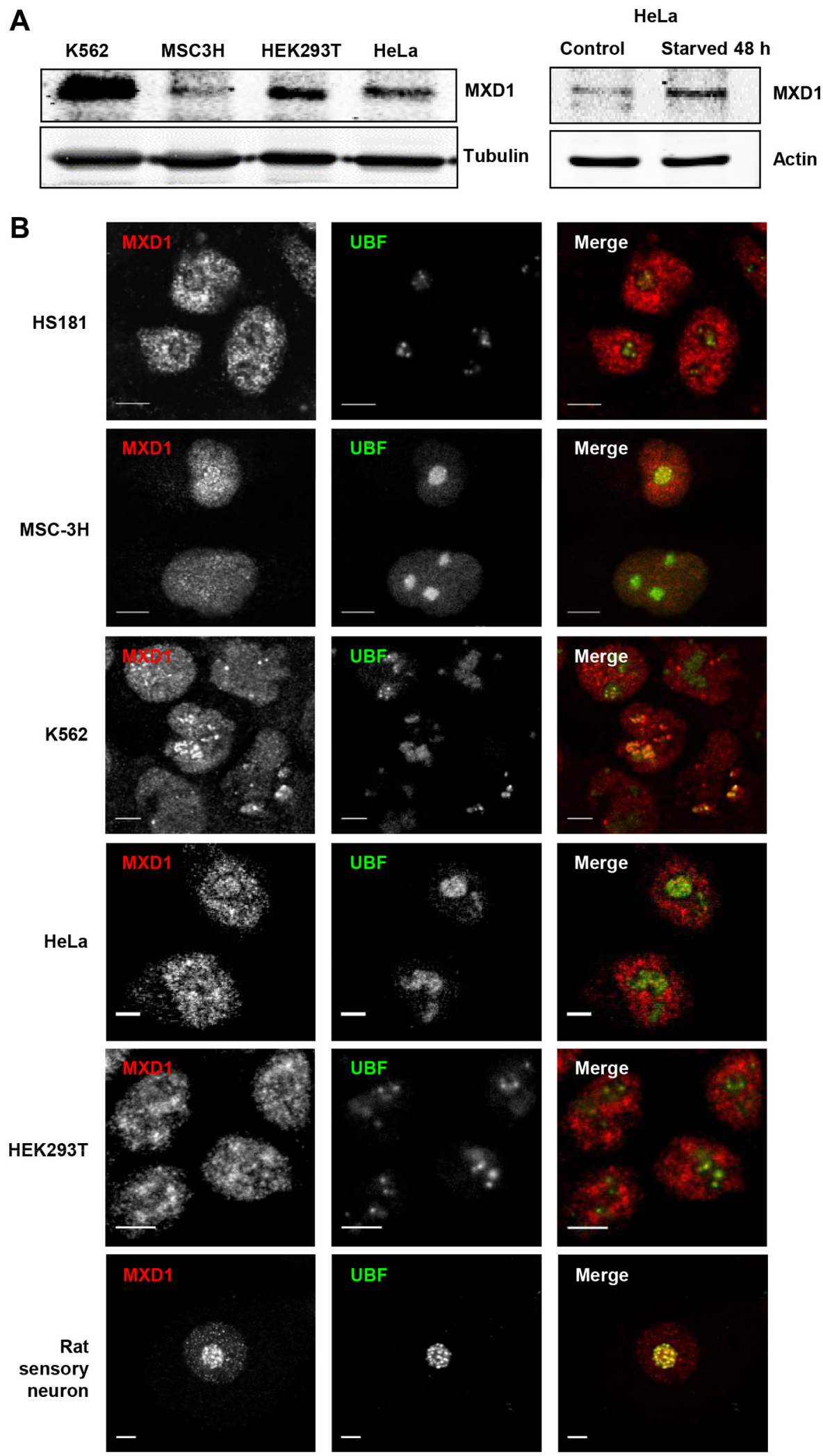

Figure 1: Endogenous MXD1 localizes in the nucleolus. (A) MXD1 protein expression in growing cells from different cell lines used in this work. MXD1 levels were analyzed by immunoblot in the indicated cell lines (left panel). The right panel shows MXD1 expression in HeLa cells deprived of serum for $48 \mathrm{~h}$. The expression of $\beta$-tubulin or $\alpha$-actin levels were determined as a control for protein loading. (B) MXD1 localization in the nucleolus. Cells from the indicated human cell lines and rat sensory neurons were double immunolabelled for MXD1 (red) and UBF (green). MXD1 showed a nuclear distribution, including the nucleolus where colocalized with UBF. Scale bars: $5 \mu \mathrm{m}$. 

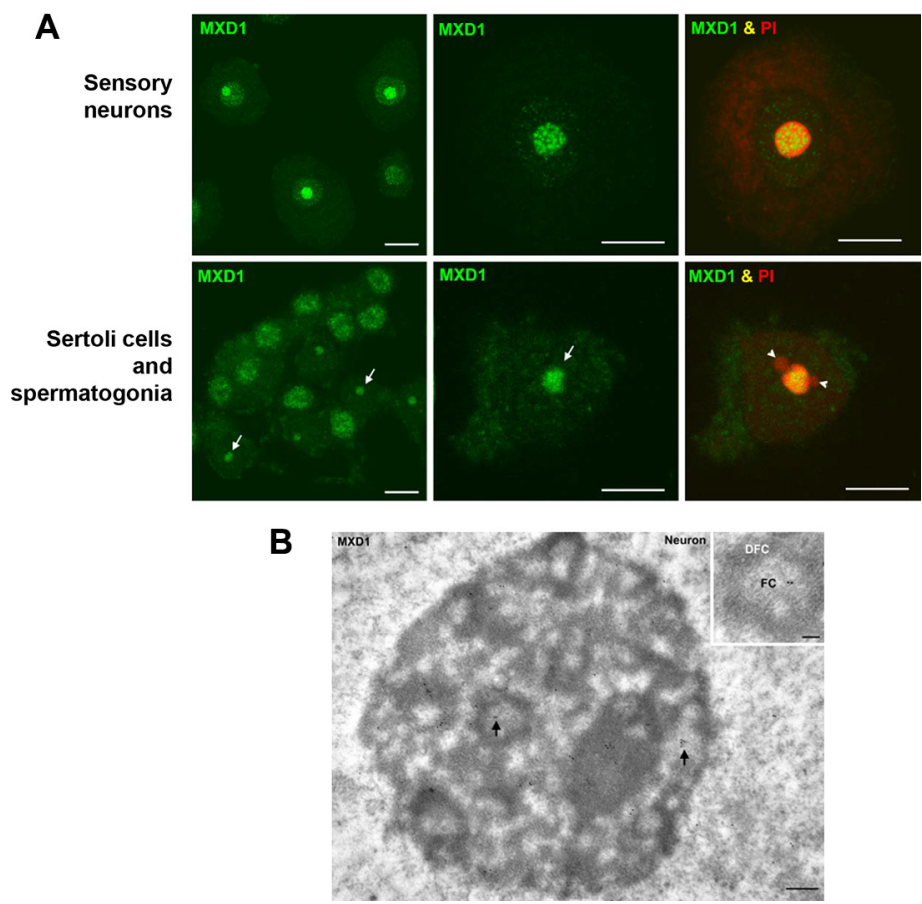

Figure 2: MXD1 expression in rat primary cells. (A) Immunofluorescence for MXD1 in sensory neurons and seminiferous tubes. In dissociated neurons from rat sensory ganglion MXD1 appeared concentrated in the nucleolus and in nucleoplasmic microfoci. Within the nucleolus, MXD1 aggregated in numerous large foci with the typical pattern of FCs. The prominent neuronal nucleolus is clearly identified by the accumulation of rRNAs stained with PI. Dissociated cells from seminiferous tubes illustrated numerous spermatogonia, with an intense nuclear signal of MXD1, and several Sertoli cells with a nucleolar pattern of MXD1 immunoreactivity (arrows). Co-staining with PI revealed the typical tripartite structure formed by the Sertoli cell nucleolus and two associated masses of perinucleolar heterochromatin (arrowheads). Scale bars: $10 \mu \mathrm{m}$. (B) Immunogold electron microscopy of MXD1 in a neuronal nucleolus. Tissue fragments from sensory ganglion were immunolabeled with the anti-MXD1 antibody and then with a secondary antibody conjugated with $10 \mathrm{~nm}$ gold particles. Immunogold particles decorated fibrillar centers (arrows) and some particles were also observed in the associated dense fibrillar component. Scale bar: $250 \mathrm{~nm}$. Inset. Higher magnification of a fibrillar center (FC) surrounded by the dense fibrillar component (DFC). Note the immunolabeling of the FC. Scale bar: $150 \mathrm{~nm}$. No immunogold particles were detected in control samples without primary antibody.

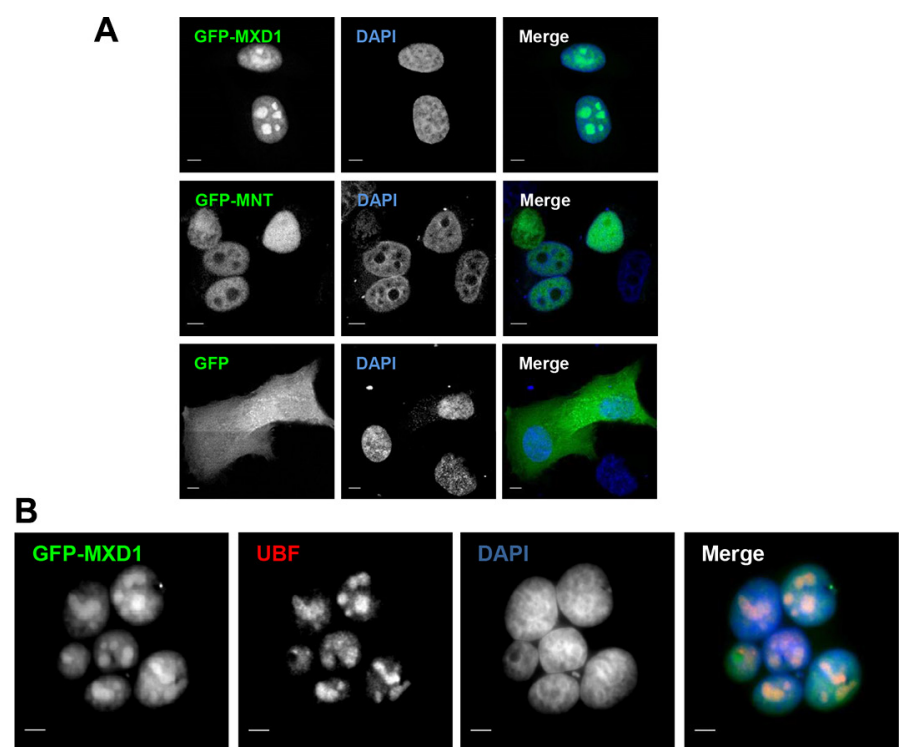

Figure 3: Ectopic MXD1 localizes in nucleoli. (A) Nucleolar localization of transfected MXD1. HeLa cells transfected with the GFP, GFP-MNT and GFP-MXD1 constructs (green). Whereas GFP-MXD1 protein localized in the nucleolus, GFP-MNT showed a nuclear staining but was absent from the nucleolus. (B) HeLa cells were transfected with the GFP-MXD1 construct and $24 \mathrm{~h}$ after transfection immunofluorescence was performed for endogenous UBF as described in Figure 1 with a secondary antibody conjugated with Texas Red. Nuclei were stained with DAPI (blue). The images were acquired by confocal (A) or epifluorescence (B) microscopy. Scale bars: $5 \mu \mathrm{m}$. 
Immunoblot analysis showed that MXD1 was present in the immunoprecipitates, indicating a UBF-MXD1 interaction (Figure 6A). Similar results were obtained in cells transfected with UBF-Flag expression vector (not shown). Next, we asked whether this interaction takes place in the nucleoli of the cells. To analyse this, we performed an in situ proximity ligation assay (PLA) in HeLa cells. As shown in Figure 6B in situ PLA signal was positive with antibodies against MXD1 and UBF. This interaction was higher in discrete areas of the nuclei, likely corresponding to the nucleoli. No interaction was detected in the cytoplasm, serving as a negative control.
Interaction was also observed between MYC and MAX (positive control), but no signal was detected when we performed the assay with antibodies against MXD1 or UBF and hemoglobins (negative controls). Signal quantification indicated that MXD1 and UBF interact but less than MYC-MAX (Figure 6C). Taken together, these results suggest that MXD1 and UBF are interacting at the site of the rRNA synthesis in the nucleolus.

As MXD1 localized in the FCs of nucleoli, we hypothesized that it might be taking part in the regulation of rRNA synthesis. We first asked whether MXD1 was bound to the rRNA genes. The human rRNA genes are organized
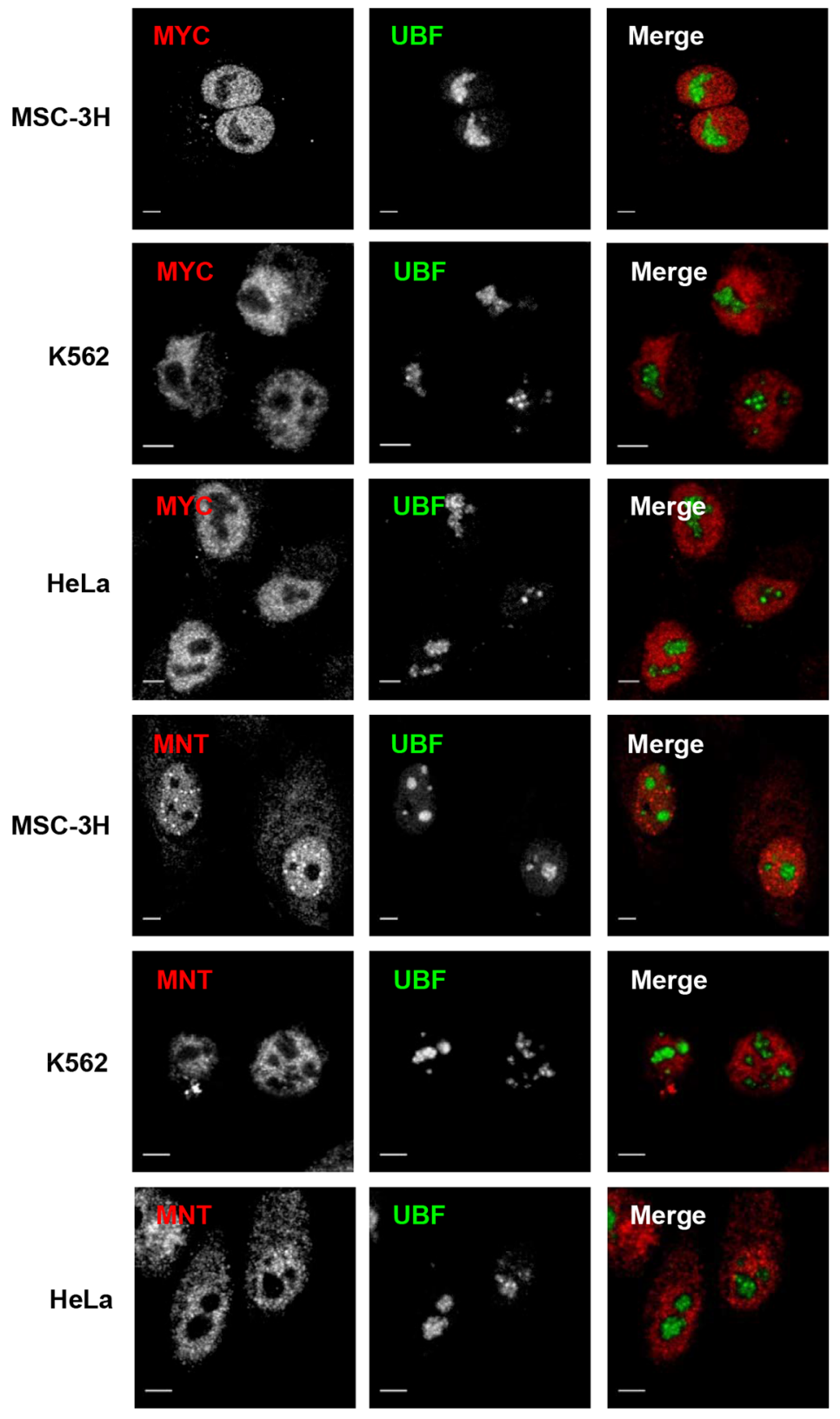

Figure 4: Nucleoplasmic but not nucleolar localization of MYC and MNT. (A) Cells from the cell lines indicate at the left were co-immunostained for UBF and MYC or MNT. Confocal merged images show the lack of co-localization of MYC and MNT with the nucleolus in all cell lines. Scale bars: $5 \mu \mathrm{m}$. 
in clusters of $\sim 43 \mathrm{~kb}$ repeats in tandem distributed among five different chromosomes (chromosome number 13, 14, 15, 21 and 22). We performed a chromatin immunoprecipitation assay (ChIP) of MXD1 on the rDNA in HeLa cells. We studied MXD1 binding to regions already analysed for MYC binding [27] in the transcribed region and in the intergenic spacer (Figure 7A). We performed this analysis in the chromatin of HeLa cells after $48 \mathrm{~h}$ of serum deprivation, in order to increase the levels of MXD1. As negative controls, we tested two amplicons mapping in the long arm of chromosomes 13 and 15 (i.e., the opposite arm to where rDNA genes map). The results showed that MXD1 was bound throughout the entire rDNA repeat, in the same regions already reported as bound to $\mathrm{MYC}[27,28]$ (Figure 7B). As a positive control, we performed ChIP analysis for UBF, which bound to the rDNA transcribed regions $(\mathrm{H} 1, \mathrm{H} 4, \mathrm{H} 8)$ and less in the IGS $(\mathrm{H} 18, \mathrm{H} 27, \mathrm{H} 42)$ [27, 29] (Figure 7B). As expected, UBF binding was much stronger than that of MXD1. Similar results were found in HEK293T cells (Supplementary Figure S3).

It is established the effect of MYC as a positive regulator of RNA pol I [27, 30-32] and it has also been

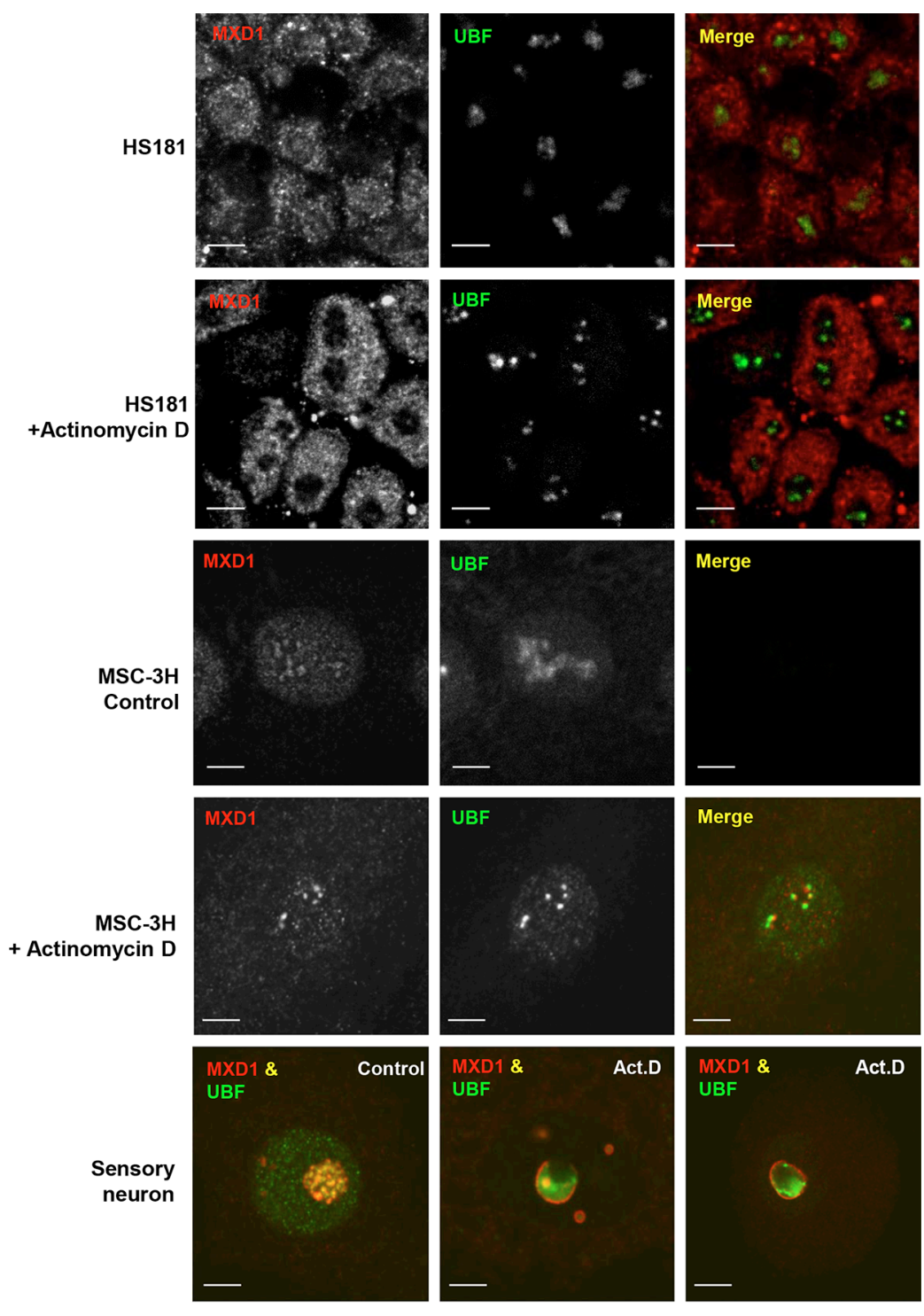

Figure 5: Nucleolar segregation of MXD1 by actinomycin D treatment. Control and actinomycin D-treated cells (50 ng/ml for $1 \mathrm{~h}$ ) from HS181 and MSC-3H cell lines were co-immunostained for MXD1 and UBF. In control HS181 and MSC-3H cells, MXD1 colocalizes with UBF in nucleoli. Inhibition of nucleolar transcription with actinomycin D induces depletion of MXD1 in HS181 nucleoli. In actinomycin D-treated MSC-3H cells, both MXD1 and UBF appear segregated at the nucleolar periphery as juxtaposed perinucleolar caps. In a control rat sensory ganglion neuron, UBF and MXD1 colocalize in numerous fibrillar centers. In contrast, fibrillar centers tend to disappear and MXD1 and UBF segregate upon systemic actinomycin D (Act.D) treatment (300 $\mu \mathrm{g} / \mathrm{k}$ for $3 \mathrm{~h}$ ). Thus, whereas MXD1 segregates as a thin ring around of both the nucleolus and smaller nucleolar fragments and colocalizes with UBF in residual fibrillar centers, the bulk of UBF tends to segregate as a large mass at the nucleolar periphery. Scale bars: $5 \mu \mathrm{m}$. 
A
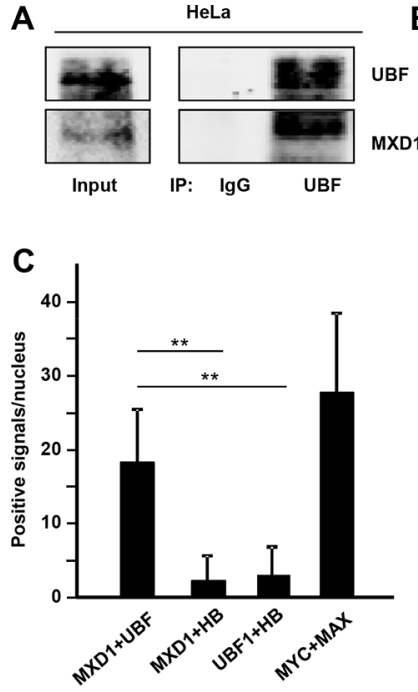
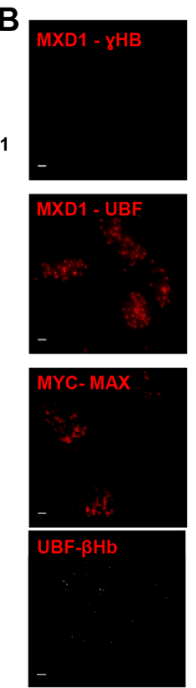
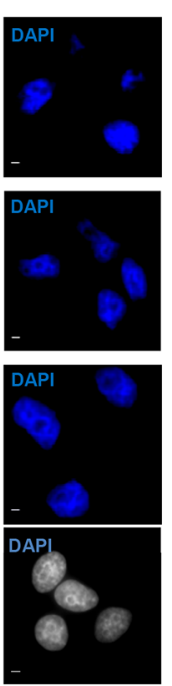
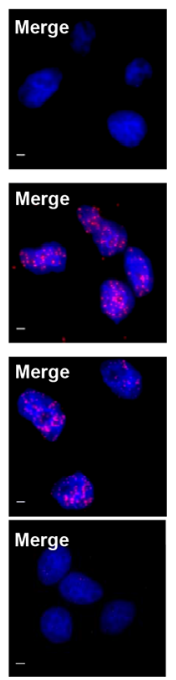

Figure 6: MXD1 and UBF interaction. (A) Co-immunoprecipitation of MXD1 and UBF in lysates of HeLa cells. Cells were serumdeprived for $48 \mathrm{~h}$ and immunoprecipitation of UBF was performed, followed by immunoblot against MXD1 and UBF. (B) In situ PLA in growing HeLa cells to test MXD1-UBF interaction. The pairs of antibodies used were anti-MXD1 and anti-UBF, anti-MYC and anti-MAX

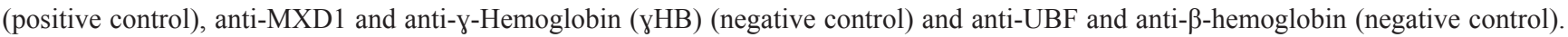
Red dots showed the MXD1-UBF interaction. DAPI staining of DNA was used to detect cell nuclei. Scale bars: $5 \mu \mathrm{m}$. (C) Quantification of PLA signals. PLA positive signals per nuclei were quantified using ImageJ software. At least 200 nuclei were counted for each experimental condition. Data are mean \pm s.e.m $* * P<0.01$

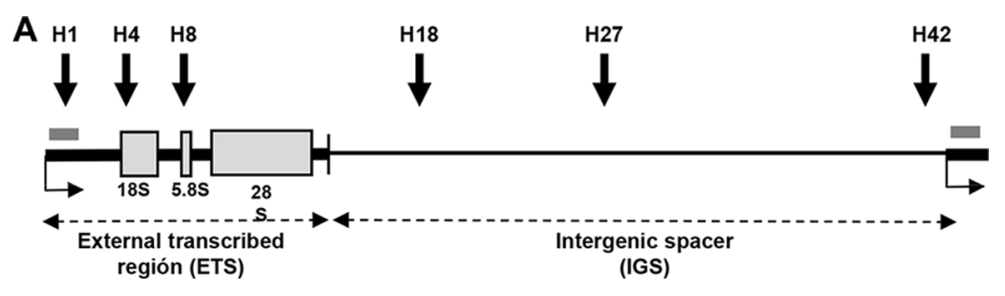

B
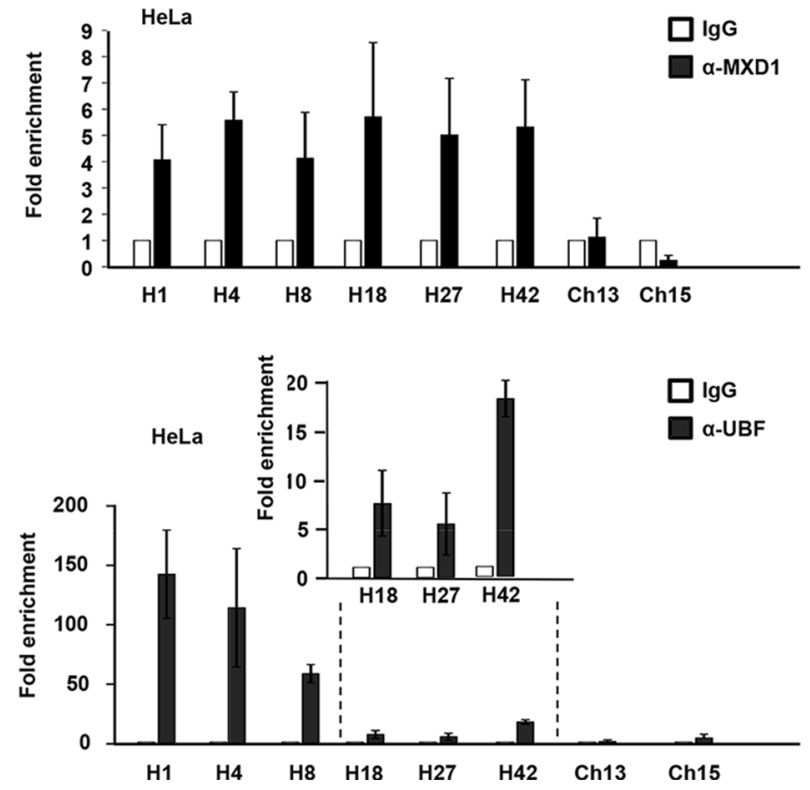

Figure 7: MXD1 binding to rDNA chromatin. (A) Schematic representation of a rDNA repeat showing the sequences of the three mature rRNAs (grey boxes), the introns (thick line) and the intergenic region (IGS, thin line). The grey bar represents the amplicon used for pre-rRNA determination by RT-qPCR. (B) ChIP of MXD1 and UBF in HeLa cells deprived of serum for $48 \mathrm{~h}$. The amplicons H1-H42 cover different regions of the rDNA gene and intergenic regions [27]. Data are mean values from four ChIP experiments. The inset shows the signals of UBF in the H18-H42 amplicons. 
reported that MXD1 down-regulates rRNA synthesis throughout the repression of UBF expression in fibroblasts [33]. We analyzed in our models whether MXD1 down-regulates rDNA transcription. We transfected the K562 cells with siRNA against MXD1 and at $72 \mathrm{~h}$ post-transfection we counted the cells and prepare protein lysates and total RNA. We confirmed a decrease in MXD1 protein levels in cells transfected with the siRNA, whereas UBF levels did not change (Figure 8A, left panel). We also tested the effect of MXD1 overexpression on UBF levels. Cell were transduced with lentivirus containing the GFP-MXD1 gene and 5 days after the levels of UBF were determined by immunoblot. The results showed no significant changes in UBF levels upon MXD1 overexpression (Figure 8A, right panel). Next, we determined the amounts of total RNA per cell in MXD1 knock-down and in the control cells. Since rRNA accounts for approximately $80 \%$ of total RNA in the cell [20], we assumed that if there was a change in total RNA levels it would be mainly due to ribosomal RNA. As shown in Figure 8B, MXD1 knock-down in K562 cells led to a higher amount of total RNA in the cell as compared with controls. This tendency was observed in the four experiments performed, although the difference with the control cells was not statistically significant $(P=0.37)$. Next we determined the levels of pre-rRNA (45S rRNA) which is the primary and unstable product of RNA pol I transcription, in MXD1-silenced K562 cells. pre-RNA was measured by RT-qPCR using 45S rRNA specific primers and the results showed higher levels of $45 \mathrm{~S}$ rRNA in cells with decreased MXD1 levels (Figure 8C). We also determined the pre-rRNA levels in HeLa cells transfected
A

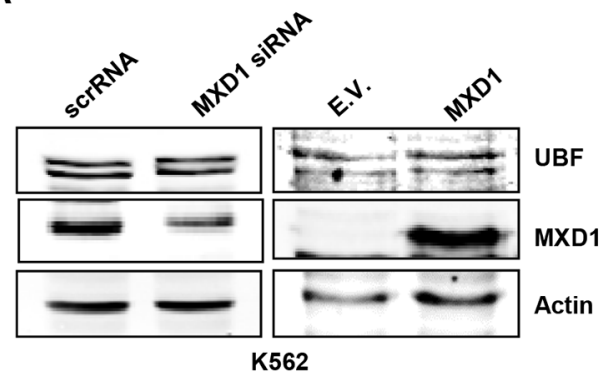

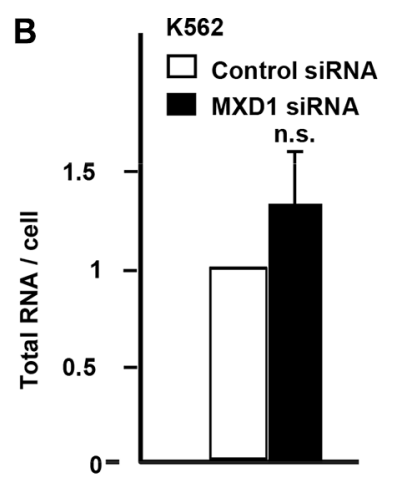

E
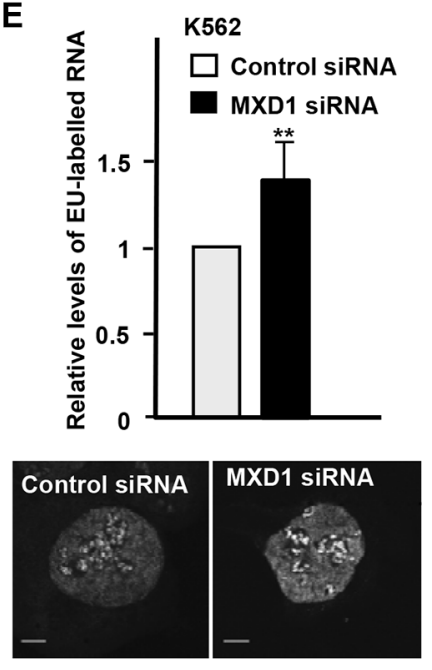

Figure 8: Effect of MXD1 depletion on rRNA levels. (A) Left panel: K562 were transfected with MXD1 siRNA or control siRNA. $72 \mathrm{~h}$ after transfection, MXD1 and UBF levels were analysed by immunoblot. Right panel: K562 were infected with GFP-MXD1 lentivirus or empty vector lentivirus (E.V.). 5 days after infection MXD1 and UBF levels were analysed by immunoblot. The levels of $\alpha$-actin were determined as a control of protein loading. (B) Total RNA per cell in K562 cells $72 \mathrm{~h}$ after MXD1-siRNA transfection. (C) Expression of 45S pre-rRNA upon MXD1 knock-down in K562 cells. Cells were transfected with MXD1 siRNA and $72 \mathrm{~h}$ after transfection the RNA was analyzed by RT-qPCR. Data are mean \pm s.e.m. from four independent transfections. (D) Expression of 45S pre-rRNA upon MXD1 knockdown in HeLa cells. Cells were transfected with a MXD1 short-hairpin vector and after 15 days of selection with puromycin, RNA was prepared and the expression of $45 \mathrm{~S}$ pre-rRNA was analyzed by RT-qPCR. Data are mean values from two independent transfections \pm s.e.m. The depletion of MXD1 protein was confirmed by immunoblot (upper panel). (E) Nascent EU-labelled rRNA after a 15 min EU pulse in K562 cells transfected with MXD1 siRNA. Signals from 100 cells were quantified using ImageJ software. Two representative cells are shown in the bottom panel. Scale bars: $5 \mu \mathrm{m}$. Error bars are s.d. ${ }^{* *} P<0.001$. 
with sh-MXD vector. The decrease in MXD1 protein was confirmed by immunoblot (Figure 8D, upper panel). The results showed an elevation of $45 \mathrm{~S}$ pre-rRNA upon MXD1 silencing (Figure 8D, lower panel). Finally, we tested the rate of the novo rRNA synthesis with pulses of 5-ethynyluridine (EU). The increased rRNA synthesis upon MXD1 depletion in K562 was also observed after labelling this EU incorporation in nascent rRNA with an azide-containing Alexa Fluor594 fluorophore (Figure 8E). In a complementary approach, we tested the effect of MXD1 enforced expression on rRNA synthesis. HeLa and HEK293T cells were transfected with a GFP-MXD1 expression vector, and MSC-3H cells were transduced with GFP-MXD1 lentiviral particles. In the three cell lines GFP-MXD1 was overexpressed $48 \mathrm{~h}$ after transfection as shown by immunoblot (Figure 9A). De novo RNA synthesis was determined by EU pulse labelling. The EU signals revealed that those cells with MXD1 overexpression showed a reduced RNA synthesis with respect to controls (Figure 9B, 9C). The EU labelling
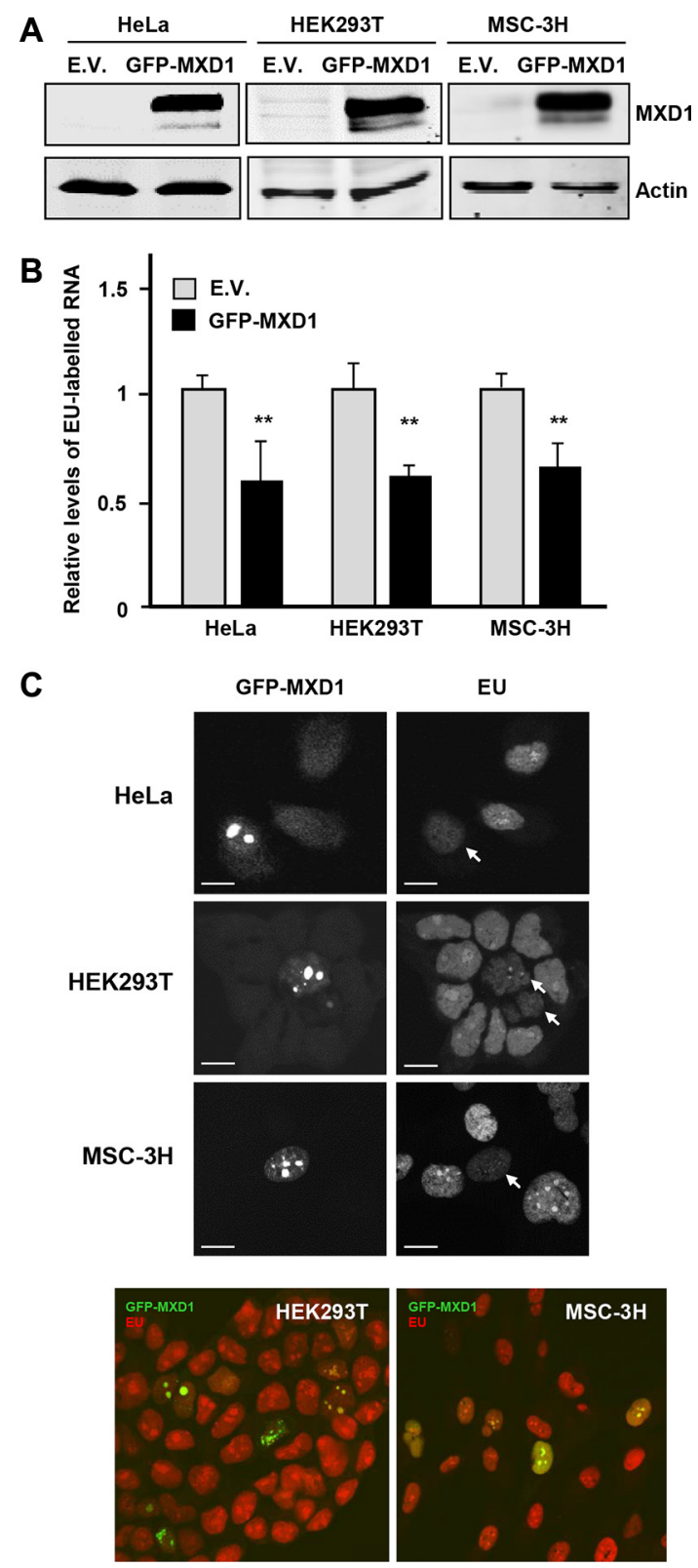

Figure 9: Effect of MXD1-enforced expression on rRNA synthesis. (A) Overexpression of GFP-MXD1 protein after transfection of GFP-MXD1 in HeLa and HEK293T, and lentiviral transduction of MSC-3H cells. The GFP-MXD1 protein was detected by immunoblot $48 \mathrm{~h}$ after transfection or virus infection. $\alpha$-actin levels were determined as a control for protein loading. (B) Nascent EU-labelled rRNA after a EU pulse of $45 \mathrm{~min}$ in the indicated cell lines transfected with an expression vector for GFP-MXD1 or the empty vector (E.V., pReceiverLv103 vector). Error bars are s.d. $* * P<0.001$. (C) Representative images showing a transfected (GFP-positive) cell and several untransfected cells. Note the accumulation of GFP-MXD1 in the nucleoli and the reduced EU labelling (arrows). Representative confocal merged images of HEK293T and MSC-3H cells showing GFP-MXD1 (green) and EU-labelled RNA (red). Scale bars: $10 \mu \mathrm{m}$. 
in the cells transfected or transduced with the empty vector was similar than in the non-transfected cells (not shown).

\section{DISCUSSION}

In this work we report that MXD1 localizes within the nucleolus and binds UBF and rDNA chromatin. These novel findings open new biological activities for MXD1. Nucleolar localization of endogenous MXD1 was observed in growing cells from different tissues origins (carcinoma, leukemia, embryonic stem cells) as well as in primary post-mitotic cells as neurons and Sertoli cells. MXD1 accumulates in the nucleolus although it is also present in the nucleoplasm. However, it is of note that MXD1 nucleolar localization was observed for endogenous MXD1 in proliferating cells, where it is expressed at relative low levels, as compared to quiescent cells. Moreover, when MXD1 levels were elevated after transfection, the MXD1 was strongly accumulated in nucleoli. In contrast, overexpressed MNT, another member of the MXD family, did not accumulate in nucleoli, supporting the specific nucleolar localization of MXD1. Previous reports have also described a nucleolar localization for MYC when expressed at supraphysiological levels or upon treatment with proteasome inhibitors [27, 31, 34]. In contrast, in our experiments we have analysed basal MYC levels in proliferating cells. In this setting MYC is present in the nucleoplasm and excluded from the nucleoli in the cell lines under analysis.

Nucleolar localization of MXD1 led us to explore the possibility of an involvement of MXD1 in rRNA synthesis, the major biological role of nucleolus. In fact, by immunofluorescence MXD1 concentrates in FCs and by immunogold electron microscopy MXD1 distributes in FCs and associated DFC, two functionally linked structures -the FC/DFC unit- where rDNA transcription and initial steps of rRNA processing take place $[17,35]$.

In fact, inhibition of RNA pol I transcription with a low concentration of actinomycin $\mathrm{D}$, which induces peripheral segregation of both the rRNA genes and UBF [36], also redistributes MXD1 at the periphery of the nucleolus. This suggests that MXD1 and UBF remain associated with inactive rRNA genes. In this vein, it has been already reported that MXD1 downregulates rRNA synthesis through MXD1-dependent downregulation of UBF in mouse fibroblasts [33]. We have confirmed the MXD1-dependent downregulation of rRNA synthesis. However, in K562 cells we could not detect a significant change in UBF levels upon MXD1 depletion or overexpression. Therefore, in our model MXD1 must impair rRNA synthesis through an alternative mechanism, although a small effect on UBF may persist. It is conceivable that MXD1 impairs UBF function in rDNA transcription through the MXD1-UBF interaction. Actually, the fact that MXD1 binds not only the promoter but also the IGS in the rDNA chromatin supports an indirect involvement of MXD1 in the rDNA transcription regulation rather than a direct repressive effect upon binding to E-boxes. So it cannot be ruled out the possibility that MXD1 binds to rDNA through another DNA-bound protein. The detailed molecular mechanisms of this interaction require further work to be unveiled.

The ability of MYC to stimulate ribosome biogenesis is critical for the orchestration of the processes of cell cycling and energy production required for proliferation of cancer cells [37]. Our work suggests that MXD1 antagonizes MYC in its positive regulation of rRNA synthesis. rRNA and ribosomal protein synthesis contribute to MYC oncogenic activity. Indeed, the inhibition of MYC activity on RNA pol I has been proposed as a therapeutic target [38]. We hypothesize that a major role of MXD1 could be to curb excessive MYC activity on ribosome biosynthesis and cell growth. Further work is required to demonstrate these hypotheses.

\section{MATERIALS AND METHODS}

\section{Cell culture transfections and lentiviral transduction}

Human K562 chronic myeloid leukemia, HEK293T embryonic kidney and HeLa cervical cancers cells, were from ATCC and grown in RPMI (K562) or DMEM (other cell lines) with fetal calf serum and antibiotics. MSC-3H cells, transformed human mesenchymal stem cells, were grown as described [39]. Human embryonic stem cells HS181 [40] were grown using mTeSR1 medium (StemCell Technologies) on Geltrex-coated plates (ThermoFisher). Transient transfections were carried out using the jetPEI reagent (Polyplus Transfection) or with Ingenio Electroporation solution (Mirus) in an Amaxa nucleofector. For lentivirus production, HEK293T cells were transfected using the jetPEI reagent method with virion packaging vectors (pCMV-VSV-G and psPAX2) and the GFP-MXD1 lentiviral construct or its empty vector in a ratio amount of 1:3:4 respectively. Two days after transfection, supernatant containing lentiviral particles was collected and filtered through $0.45 \mu \mathrm{m}$ pore size filters (Merck Millipore). Viral particles were concentrated by precipitation using PEG8000 and resuspended in serumfree DMEM. K562 and MSC-3H cells were infected with a MOI of 2 in presence of $5 \mu \mathrm{g} / \mathrm{ml}$ of polybrene for $48 \mathrm{~h}$. The plasmids and siRNAs used are described in Supplementary Table S1

\section{Immunoblot and immunoprecipitation}

Cell lysis, immunoblots and immunoprecipitations were performed as described elsewhere [41]. The primary antibodies used are described in Supplementary Table S2 
and the immunoblots were revealed with an Odyssey scanner (Li-Cor Biosciences).

\section{Immunofluorescence and immunogold staining}

Tissue fragments from rat testes and trigeminal ganglion were fixed with $4 \%$ paraformaldehyde in PBS. Squash preparations from both tissues were processed and immunostained as reported previously [23]. The protocol was approved by the Ethics Committee of the University of Cantabria. Adherent cells grown on glass coverslips and were fixed with $4 \%$ paraformaldehyde in PBS for $15 \mathrm{~min}$ at room temperature. Fixed cells were washed with PBS and permeabilized with $1 \%$ Triton X-100 in PBS during $30 \mathrm{~min}$. Then, cells were treated with blocking buffer (3\% BSA; 0.1\% Triton X-100 in PBS) for $20 \mathrm{~min}$, washed with PBS and $0.1 \%$ Triton X-100 in PBS, and incubated overnight at $4^{\circ} \mathrm{C}$ with the primary antibodies diluted in blocking buffer. The slides were incubated for $1 \mathrm{~h}$ at room temperature with the secondary antibody conjugated with FITC or Cy3 (Jackson Laboratories). The samples were mounted with ProLong Gold Antifade mountant (LifeTechnologies). Confocal images were obtained with a Leica TCS SP5 microscope and processed using Adobe Photoshop and ImageJ (http://rsb.info.nih. gov/ij/) softwares. For immunogold electron microscopy, tissue fragments from sensory ganglion were fixed with $4 \%$ paraformaldehyde and embedded in Lowicryl $4 \mathrm{M}$. Ultrathin sections were immunolabeled with the antiMXD1 antibody and then with a secondary antibody conjugated with $10 \mathrm{~nm}$ gold particles The antibodies used are described in the Supplementary Table S2.

\section{In situ proximity ligation assay}

In situ Proximity Ligation Assay (PLA) was performed with Duolink in situ Red Starter kit Mouse/ Rabbit (Sigma-Aldrich) according to manufacturer's instructions with home-made buffers as previously described [41]. In situ PLA positive signals were quantified using the ImageJ software. At least 200 nuclei were measured for each experimental condition. Cell samples were visualized using a confocal microscope as described above. The primary antibodies used are described in Supplementary Table S2.

\section{RNA analysis}

Total RNA was isolated using the RNeasy kit (Qiagen). cDNA was generated by reverse transcription (RT) using the iScript (Bio-Rad). Quantitative polymerase chain reaction (qPCR) was performed with specific primers (Supplementary Table S3) using the iQSYBR Green supermix (Bio-Rad). Labelling of rRNA with 5-ethynyluridine (EU) was performed with the Click-iT
RNA AlexaFluor594 kit (ThermoFisher) for 15 min (K562 cells) or $50 \mathrm{~min}$ (HeLa, HEK293T and MSC-3H cells) and assessed by microscopic imaging. Levels of mRNA and pre-rRNA were normalized against actin and RPS14 mRNA levels.

\section{Chromatin immunoprecipitation}

Chromatin immunoprecipitation (ChIP) was performed essentially as described [41]. Immunoprecipitated DNA was purified with the QIAquick PCR Purification Kit (Qiagen) and analysed by qPCR. The antibodies and primers used are described in Table 2 and Table 3 respectively.

\section{ACKNOWLEDGMENTS}

We thank Outi Hovatta, Pablo Menéndez and René Rodríguez for reagents and cell lines, Elsa Valdizán, Fuencisla Pilar and Victor Campa for advice with histology and microscopy techniques, and M. Dolores Delgado for helpful discussions

\section{CONFLICTS OF INTEREST}

The authors declare no competing or financial interests

\section{GRANT SUPPORT}

The work was supported by grants SAF2014-53526 (to JL), BFU2014-54754P (to ML and MTB) from Spanish Economy and Competitiveness Ministry (MINECO), and grants RETIC-RD012-036-033 (to JL) and CIBERNED CB06/05/0037 (to ML) from Instituto Carlos III to JL. These funding were co-sponsored by the FEDER program. MCL was recipient of a fellowship from the FPU program from MINECO.

\section{Authors' contributions}

JL conceived the study. JL, ML and MB wrote the manuscript. MCLN and RB carried out most experiments, JMG and OT carried out the experiments of Figures 5 and 9, LGG carried out some experiments of Figure 8, EGA performed some experiments with the stem cells. ML and MB prepared most figures with confocal images. All authors reviewed the results and approved the final version of the manuscript.

\section{REFERENCES}

1. Ayer DE, Kretzner L, Eisenman RN. Mad: a heterodimeric partner for Max that antagonizes Myc transcriptional activity. Cell. 1993; 72:211-222. 
2. Meyer N, Penn LZ. Reflecting on 25 years with MYC. Nat Rev Cancer. 2008; 8:976-990.

3. Dang CV. MYC on the path to cancer. Cell. 2012; 149:22-35.

4. Conacci-Sorrell M, McFerrin L, Eisenman RN. An Overview of MYC and Its Interactome. Cold Spring Harb Perspect Med. 2014; 4:1-24.

5. Ge Z, Li W, Wang N, Liu C, Zhu Q, Bjorkholm M, Gruber A, Xu D. Chromatin remodeling: recruitment of histone demethylase RBP2 by Mad1 for transcriptional repression of a Myc target gene, telomerase reverse transcriptase. FASEB J. 2010; 24:579-586.

6. Luscher B. MAD1 and its life as a MYC antagonist: an update. Eur J Cell Biol. 2012; 91:506-514.

7. Zhou ZQ, Hurlin PJ. The interplay between Mad and Myc in proliferation and differentiation. Trends Cell Biol. 2001; 11:S10-14.

8. Hurlin PJ, Foley KP, Ayer DE, Eisenman RN, Hanahan D, Arbeit JM. Regulation of Myc and Mad during epidermal differentiation and HPV- associated tumorigenesis. Oncogene. 1995; 11:2487-2501.

9. Chin L, Schreiber-Agus N, Pellicer I, Chen K, Lee HW, Dudast M, Cordon-Cardo C, DePinho RA. Contrasting roles for Myc and Mad proteins in cellular growth and differentiation. Proc Natl Acad Sci USA. 1995; 92:8488-8492.

10. Lahoz EG, Xu L, Schreiber-Agus N, DePinho RA. Suppression of Myc, but not E1a, transformation activity by Max- associated proteins, Mad and Mxi1. Proc Natl Acad Sci USA. 1994; 91:5503-5507.

11. Hurlin PJ, Ayer DE, Grandori C, Eisenman RN. The Max transcription factor network: involvement of Mad in differentiation and an approach to identification of target genes. Cold Spring Harb Symp Quant Biol. 1994; 59:109-116.

12. Iritani BM, Delrow J, Grandori C, Gomez I, Klacking M, Carlos LS, Eisenman RN. Modulation of T-lymphocyte development, growth and cell size by the Myc antagonist and transcriptional repressor Mad1. Embo J. 2002; 21:4820-4830.

13. Hernandez-Verdun D, Roussel P, Thiry M, Sirri V, Lafontaine DL. The nucleolus: structure/function relationship in RNA metabolism. Wiley Interdiscip Rev RNA. 2010; 1:415-431.

14. Schwarzacher HG, Wachtler F. The functional significance of nucleolar structures. Ann Genet. 1991; 34:151-160.

15. Scheer U, Hock R. Structure and function of the nucleolus. Curr Opin Cell Biol. 1999; 11:385-390.

16. McStay B, Grummt I. The epigenetics of rRNA genes: from molecular to chromosome biology. Annu Rev Cell Dev Biol. 2008; 24:131-157.

17. Koberna K, Malinsky J, Pliss A, Masata M, Vecerova J, Fialova M, Bednar J, Raska I. Ribosomal genes in focus: new transcripts label the dense fibrillar components and form clusters indicative of "Christmas trees" in situ. J Cell Biol. 2002; 157:743-748.
18. Cheutin T, O'Donohue MF, Beorchia A, Vandelaer M, Kaplan H, Defever B, Ploton, Thiry M. Three-dimensional organization of active rRNA genes within the nucleolus. J Cell Sci. 2002; 115:3297-3307.

19. Boisvert FM, van Koningsbruggen S, Navascues J, Lamond AI. The multifunctional nucleolus. Nat Rev Mol Cell Biol. 2007; 8:574-585.

20. Moss T, Langlois F, Gagnon-Kugler T, Stefanovsky V, A housekeeper with power of attorney: the rRNA genes in ribosome biogenesis. Cell Mol Life Sci. 2007; 64:29-49.

21. Hurlin PJ, Queva C, Koskinen PJ, Steingrimsson E, Ayer DE, Copeland NG, Jenkins NA, Eisenman RN. Mad3 and Mad4: novel Max-interacting transcriptional repressors that suppress c-myc dependent transformation and are expressed during neural and epidermal differentiation. Embo J. 1996; 15:2030.

22. Zhu J, Blenis J, Yuan J. Activation of PI3K/Akt and MAPK pathways regulates Myc-mediated transcription by phosphorylating and promoting the degradation of Mad1. Proc Natl Acad Sci USA. 2008; 105:6584-6589.

23. Casafont I, Bengoechea R, Navascues J, Pena E, Berciano MT, Lafarga M. The giant fibrillar center: a nucleolar structure enriched in upstream binding factor (UBF) that appears in transcriptionally more active sensory ganglia neurons. J Struct Biol. 2007; 159:451-461.

24. Perry RP, Kelley DE. Inhibition of RNA synthesis by actinomycin D: characteristic dose-response of different RNA species. J Cell Physiol. 1970; 76:127-139.

25. Torrano V, Navascues J, Docquier F, Zhang R, Burke LJ, Chernukhin I, Farrar D, Leon J, Berciano MT, Renkawitz R, Klenova E, Lafarga M, Delgado MD. Targeting of CTCF to the nucleolus inhibits nucleolar transcription through a poly(ADP-ribosyl)ation-dependent mechanism. J Cell Sci. 2006; 119:1746-1759.

26. Journey LJ, Goldstein MN. Electron microscope studies on HeLa cell lines sensitive and resistant to actinomycin D. Cancer Res. 1961; 21:929-932.

27. Grandori C, Gomez-Roman N, Felton-Edkins ZA, Ngouenet C, Galloway DA, Eisenman RN, White RJ. c-Myc binds to human ribosomal DNA and stimulates transcription of rRNA genes by RNA polymerase I. Nat Cell Biol. 2005; 7:311-318.

28. Shiue CN, Nematollahi-Mahani A, Wright AP. Myc-induced anchorage of the rDNA IGS region to nucleolar matrix modulates growth-stimulated changes in higher-order rDNA architecture. Nucleic Acids Res. 2014; 42:5505-5517.

29. O'Sullivan AC, Sullivan GJ, McStay B, UBF binding in vivo is not restricted to regulatory sequences within the vertebrate ribosomal DNA repeat. Mol Cell Biol. 2002; 22:657-668.

30. Campbell KJ, White RJ. MYC regulation of cell growth through control of transcription by RNA polymerases I and III. Cold Spring Harb Perspect Med. 2014; 4. 
31. Arabi A, Wu S, Ridderstrale K, Bierhoff H, Shiue C, Fatyol K, Fahlen S, Hydbring P, Soderberg O, Grummt I, Larsson LG, Wright AP. c-Myc associates with ribosomal DNA and activates RNA polymerase I transcription. Nat Cell Biol. 2005; 7:303-310.

32. Schlosser I, Holzel M, Murnseer M, Burtscher $H$, Weidle UH, Eick D. A role for c-Myc in the regulation of ribosomal RNA processing. Nucleic Acids Res. 2003; 31:6148-6156.

33. Poortinga G, Hannan KM, Snelling H, Walkley CR, Jenkins A, Sharkey K, Wall M, Brandenburger Y, Palatsides M, Pearson RB, McArthur GA, Hannan RD. MAD1 and c-MYC regulate $\mathrm{UBF}$ and $\mathrm{rDNA}$ transcription during granulocyte differentiation. EMBO J. 2004; 23:3325-3335.

34. Grewal SS, Li L, Orian A, Eisenman RN, Edgar BA. Mycdependent regulation of ribosomal RNA synthesis during Drosophila development. Nat Cell Biol. 2005; 7:295-302.

35. Smirnov E, Hornacek M, Kovacik L, Mazel T, Schrofel A, Svidenska S, Skalnikova M, Bartova E, Cmarko D. Raska I, Reproduction of the FC/DFC units in nucleoli. Nucleus. 2016; 7:203-215.

36. Jordan P, Mannervik M, Tora L, Carmo-Fonseca M. In vivo evidence that TATA-binding protein/SL1 colocalizes with UBF and RNA polymerase I when rRNA synthesis is either active or inactive. J Cell Biol. 1996; 133:225-234.
37. van Riggelen J, Yetil A, Felsher DW. MYC as a regulator of ribosome biogenesis and protein synthesis. Nat Rev Cancer. 2010; 10:301-309.

38. Poortinga G, Quinn LM, Hannan RD. Targeting RNA polymerase I to treat MYC-driven cancer. Oncogene. 2015; 34:403-412.

39. Funes JM, Quintero M, Henderson S, Martinez D, Qureshi U, Westwood C, Clements MO, Bourboulia D, Pedley RB, Moncada S, Boshoff C. Transformation of human mesenchymal stem cells increases their dependency on oxidative phosphorylation for energy production. Proc Natl Acad Sci USA. 2007; 104:6223-6228.

40. Hovatta O, Mikkola M, Gertow K, Stromberg AM, Inzunza J, Hreinsson J, Rozell B, Blennow E, Andang M, Ahrlund-Richter L. A culture system using human foreskin fibroblasts as feeder cells allows production of human embryonic stem cells. Hum Reprod. 2003; 18:1404-1409.

41. Garcia-Sanz P, Quintanilla A, Lafita MC, Moreno-Bueno G, Garcia-Gutierrez L, Tabor V, Varela I, Shiio Y, Larsson LG, Portillo F, Leon J. Sin3b interacts with myc and decreases myc levels. J Biol Chem. 2014; 289:22221-22236. 